\title{
A New Kind of Vacuum Tube ----- for Proton Driver
}

\author{
Zhijing Tang \\ September, 2002
}

In Proton Driver Study II (PD2), a new technique is proposed by Weiren Chou to manufacture the vacuum tube. Basically, this smaller metal vacuum tube will be used to replace the ceramic vacuum tube, saving magnetic space. Therefore the required magnets can be made smaller, and the operation cost can be reduced. In this engineering note we investigate the structural strength and displacement of the tube, the magnetic field inside the tube and the power loss.

\section{The Vacuum Tube}

The cross section of the vacuum tube is 6 inch by 4 inch oval with thickness of 0.008 inch. The material is Inconel 718. The laser precision metal deposition (PMD) method will be used to put a spiral rib (same material with 18 mils height and 28 mils width can be applied repeatedly) around the tube to increase its strength against buckling. We select 10 layers of reinforcement (hence the total height of the rib is $0.18 \mathrm{inch}$ ) and the pitch of the rib is set to 1 inch.

Some relevant material properties of Inconel 718 are: tension modules $E=29 \mathrm{e} 6$ psi, yield strength $\sigma_{\mathrm{y}}=171 \mathrm{e} 3 \mathrm{psi}$, ultimate strength $\sigma_{\mathrm{u}}=196 \mathrm{e} 3$ psi, electric resistivity $\lambda=$ $125 \mathrm{e}-8$ ohm-m,

\section{External Magnetic Field}

Applied external magnetic field is (see Appendix I)

$$
B^{e x t}=0.85-0.632 \cos (\omega t)+0.079 \sin (2 \omega t)
$$

Where $\omega=2 \pi f$ and $f=15 \mathrm{~Hz}$. This is plotted in Fig. 1 together with its time derivative for one period. The maximum and minimum of the magnetic field is $15 \mathrm{~T}$ and $0.2 \mathrm{~T}$, respectively.

\section{Structural Model}

Strictly speaking, the spirally reinforced tube is not symmetric about any plane. To simplify the calculation, we assume that the vacuum tube is approximately symmetric about coordinate planes. Therefore we model only one quarter of a typical segment. The 
only load considered here is the vacuum pressure of 14.5 psi (electric-magnetic load is not considered here, since it is much smaller compared with vacuum pressure, see Appendix IV).

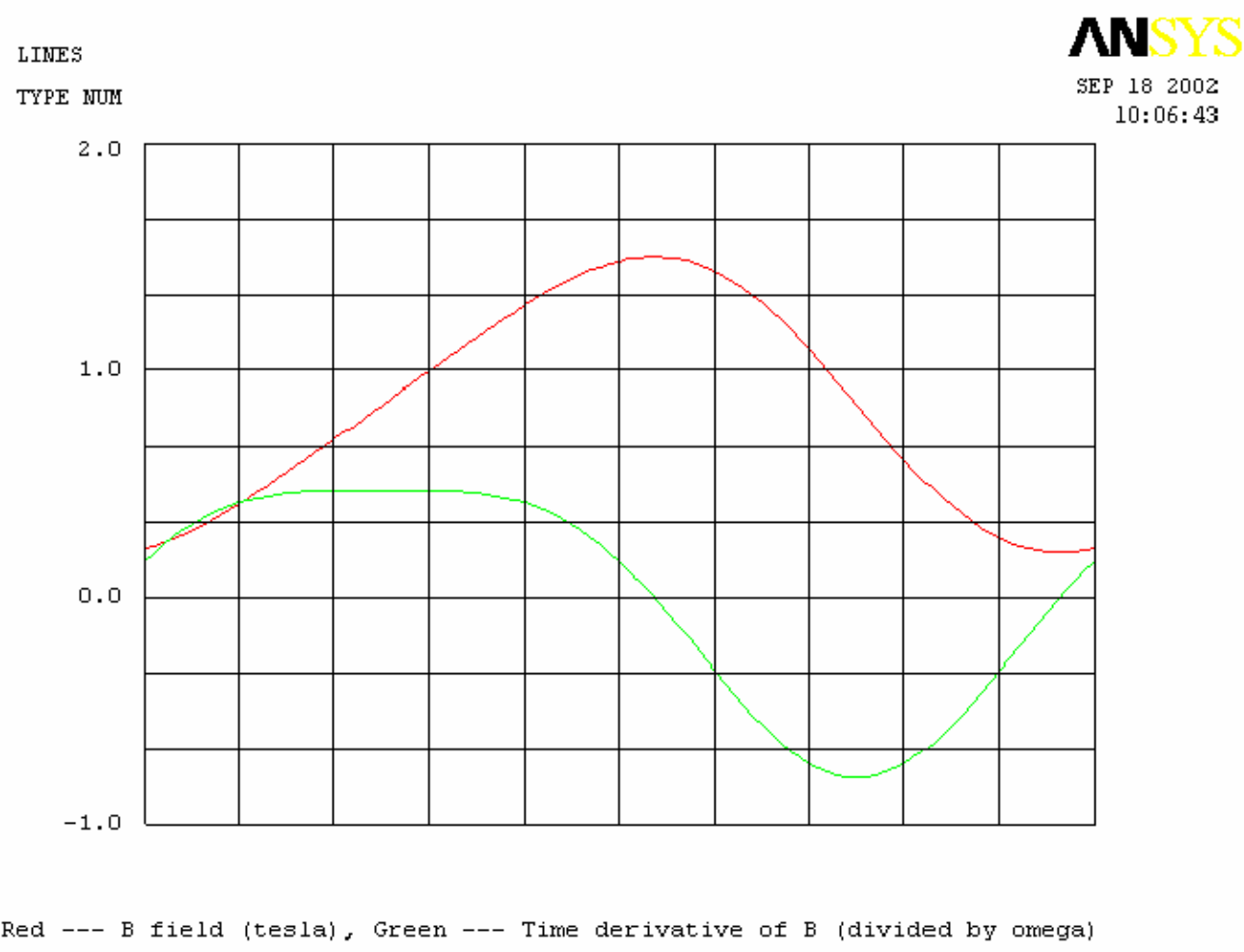

Fig.1 External magnetic field and its time derivative 


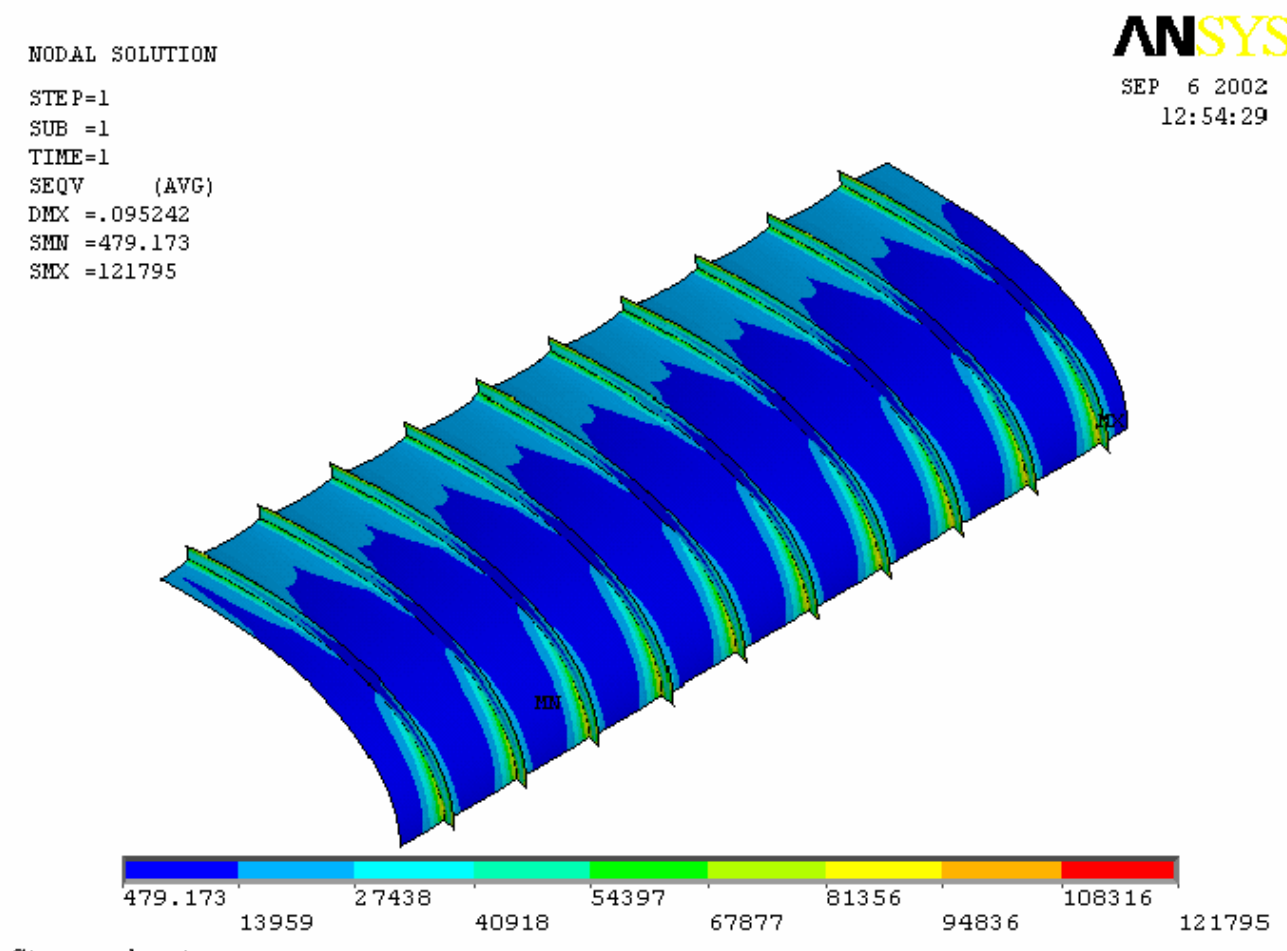

Stress due to vacuum pressure

Fig. 2 Stress plot. The maximum stress is $122 \mathrm{ksi}$.

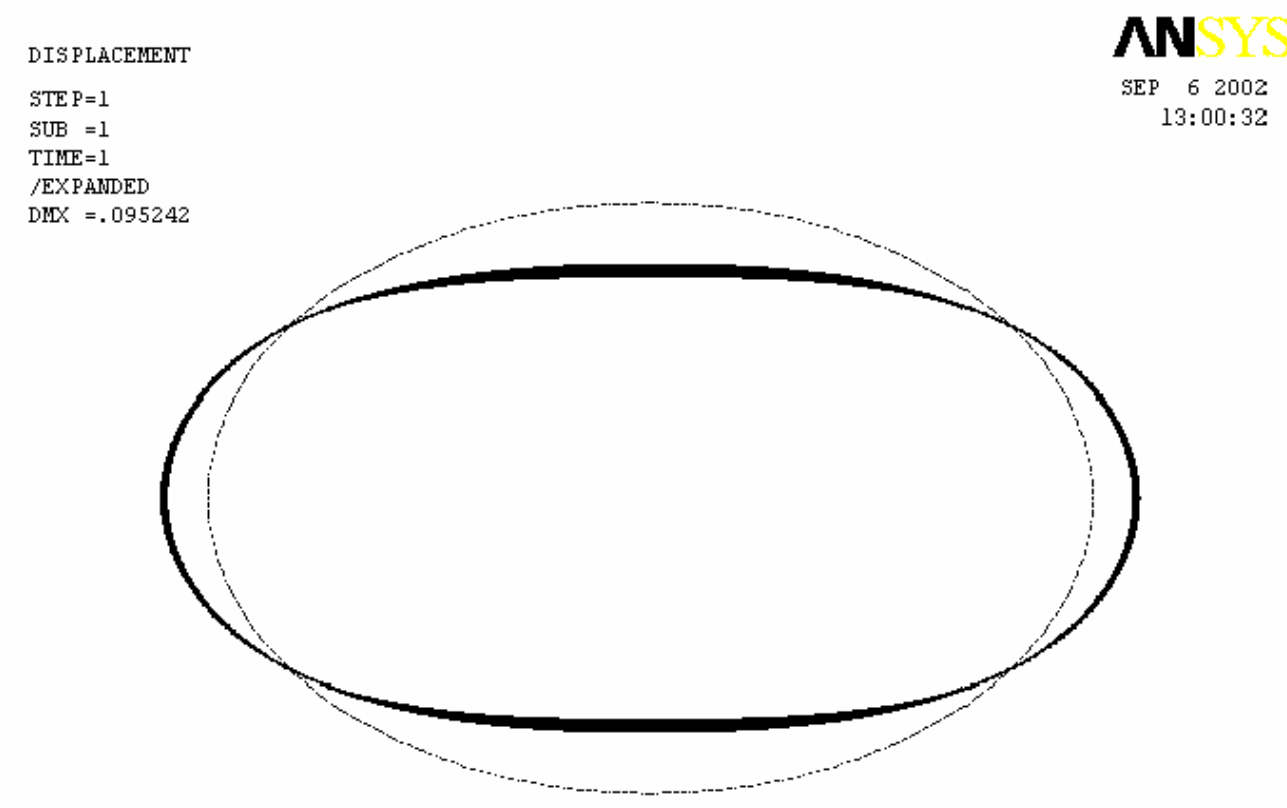

Deformed vacuum tube

Fig.3 Deformation of Vacuum Tube under vacuum pressure 
Fig. 2 plots the equivalent stress, with the maximum value of $122 \mathrm{ksi}$. This is a large number. But since our material has high yield stress and ultimate stress, this number will be alright (See Appendix VI). Fig.3 is the deformed cross section versus original shape. The major axis extended 0.061 in in both directions (about 2\%) while the minor axis shrank 0.095 in in both direction (5\%). To achieve required tube shape under vacuum condition, the tube can be made with smaller major radius and larger minor radius.

\section{Electro-Magnetic Model}

Since the vacuum tube is made of metal, time varying external magnetic field will induce eddy current. This eddy current will have two effects: distort the external field and cause power loss.

For a single spiral rib, an argument based on symmetry will lead to the conclusion that the eddy current caused by the spiral rib is negligible compared with the vacuum tube itself (see Appendix II).

We model only the vacuum tube without the reinforce rib. Use 3-d element, we made a quarter model. The model length is 1 inch. The inside and outside are filled with vacuum

and air elements. Since the effect is linear, we can analyze the $15 \mathrm{~Hz}$ field and $30 \mathrm{~Hz}$ field separately. The constant field has no eddy current effect.

\section{Power Loss}

For $15 \mathrm{~Hz}$ field, $B_{1}=0.632 \mathrm{~T}$, calculated power loss is $1.957 \mathrm{w}$, for $30 \mathrm{~Hz}$ field, $B_{2}=$ $0.632 / 8 \mathrm{~T}$, power loss is $0.122 \mathrm{w}$. Add together, we have $2.079 \mathrm{w}$ power loss for our model. Remember our model is a quarter model, so the total power loss should be 8.316 w/in, or $327 \mathrm{w} / \mathrm{m}$. This number compare well with the analytical result (see Appendix III).

\section{Magnetic Field}

Now consider the magnetic field inside the vacuum tube on $y=0$ plane. The results are plotted in following 4 figures (Figs. 4 7). From these results we see that the magnitudes are almost exactly the same as that of the applied external fields, and the lag angles are extremely small. Therefore, no correction will be needed. (See Appendix V, where we show that the magnetic field induced by the eddy current is about one thousands of the external field in magnitude.)

\section{Conclusions}

From this study, we know that the proposed vacuum tube should work, at least in theory. The problems will occur during manufacture process, there the imperfections will be introduced, even some degree of damage to the tube material will be unavoidable. Since this tube is so thin, the final strength of the vacuum tube will completely depend on the perfection of the manufacture techniques, therefore prototype study is needed. 
$(x 10 * *-1)$

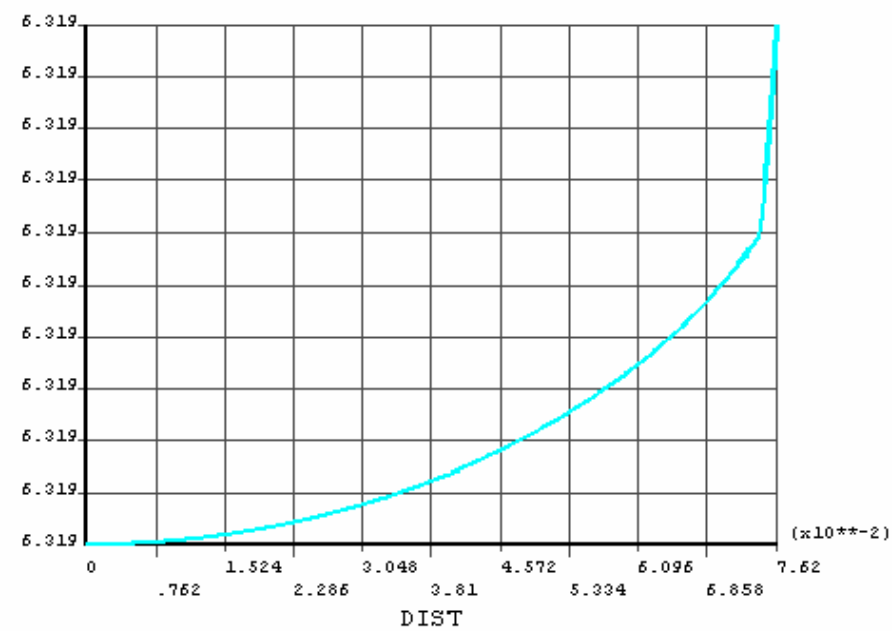

Magnetic field at $y=0$ plane, Magnitude of $15 \mathrm{~Hz}$

Fig.4 Magnetic field magnitude of the $15 \mathrm{~Hz}$ component.

POST1

NN

STE $\mathrm{P}=2$

SUB $=1$

SEP 52002

FRE $Q=30$

PATH PLOT

NOD $1=40288$

NOD $2=40001$

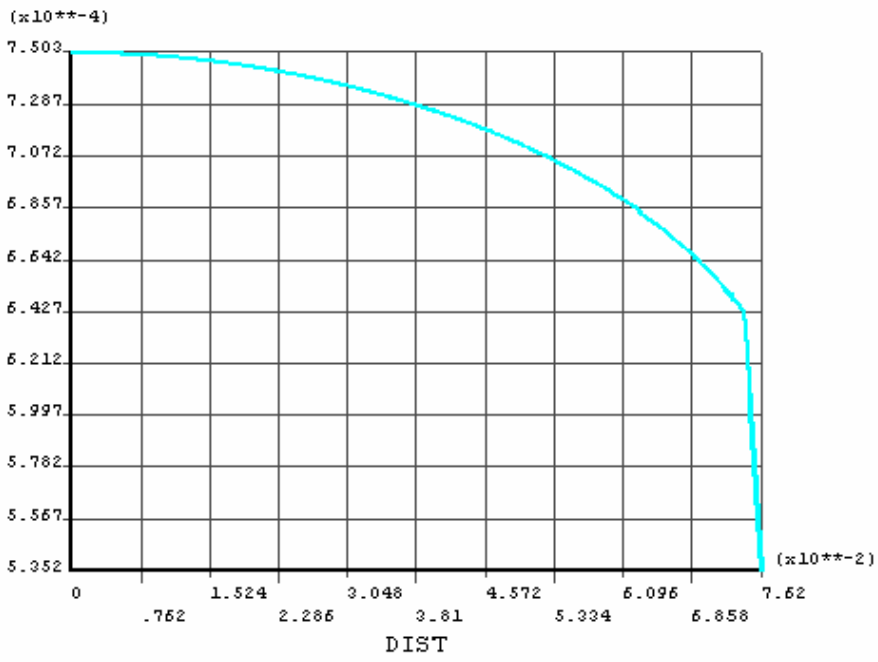

Magnetic field at $y=0$ plane, lag angle of $15 \mathrm{~Hz}$

Fig.5 Magnetic field lag angle of the $15 \mathrm{~Hz}$ component 
P0ST1

STE $P=2$

SUB $=1$

$\operatorname{FRE} Q=30$

PATH PLOT

NOD $1=40288$

NOD $2=40001$

B2
NN

SEP 52002 $16: 28: 42$

Magnetic field at $y=0$ plane, Magnitude of $30 \mathrm{~Hz}$

Fig. 6 Magnetic field magnitude of the $30 \mathrm{~Hz}$ component

P0ST1

$\mathbf{N}$

STE $\mathrm{P}=2$

FRE $0=30$

PATH PLOT

NOD $1=40288$

( $\times 10 * *-2)$

7.89

7.8

7.89

7.

7.8

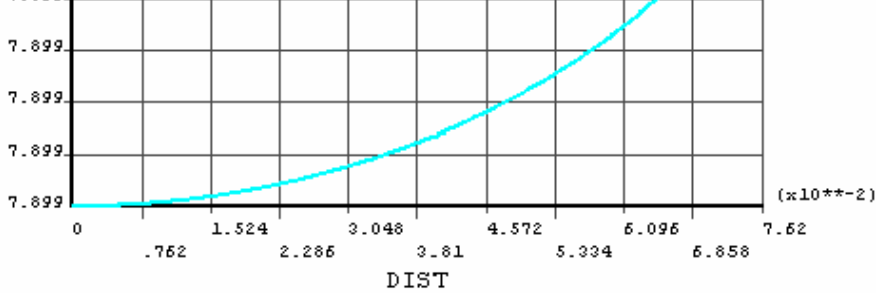

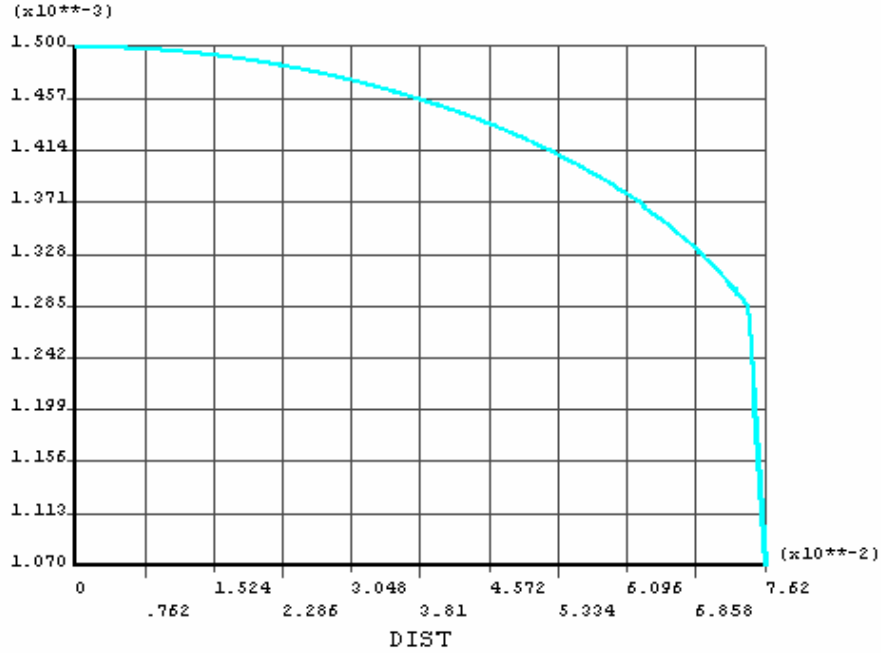

Magnetic field at $\mathrm{y}=0$ plane, lag angle of $30 \mathrm{~Hz}$

Fig. 7 Magnetic field lag angle of the $30 \mathrm{~Hz}$ component 


\section{Appendix I. The External Magnetic Field}

The external magnetic field is given in following form

$B^{e x t}=B_{0}^{e x t}+B_{1}^{e x t} \cos (\omega t)+B_{2}^{e x t} \sin (2 \omega t)$

We Know that $B_{2}^{\text {ext }}=-0.125 B_{1}^{\text {ext }}, \quad \operatorname{Max}\left\{B^{\text {ext }}\right\}=1.5 \mathrm{~T}, \quad \operatorname{Min}\left\{B^{\text {ext }}\right\}=0.2 \mathrm{~T}$.

Take the derivative of above equation, we have $\frac{\mathrm{d} B^{\text {ext }}}{\mathrm{d} t}=-B_{1}^{\text {ext }} \omega\{\sin (\omega t)+0.25 \cos (2 \omega t)\}$

Set this to zero, we get

$$
\begin{aligned}
& \sin (\omega t)=-(\sqrt{1.5}-1)=-0.22474487 \\
& (\omega t)_{1}=-2.91491145, \quad(\omega t)_{2}=-0.22668120 .
\end{aligned}
$$

Substitute back into the equation, we have

$$
\begin{aligned}
& B^{\text {ext }}=B_{0}^{\text {ext }}+B_{1}^{\text {ext }} \cos \left\{(\omega t)_{1}\right\}+B_{2}^{\text {ext }} \sin \left\{2(\omega t)_{1}\right\} \\
& =B_{0}^{e x t}+B_{1}^{e x t}(0.97441764+0.125 * 0.43799073) \\
& =B_{0}^{\text {ext }}+1.02916648 B_{1}^{\text {ext }}=\operatorname{Min}\left\{B^{\text {ext }}\right\}=0.2 \mathrm{~T} \\
& B^{\text {ext }}=B_{0}^{\text {ext }}+B_{1}^{\text {ext }} \cos \left\{(\omega t)_{2}\right\}+B_{2}^{\text {ext }} \sin \left\{2(\omega t)_{2}\right\} \\
& =B_{0}^{\text {ext }}+B_{1}^{\text {ext }}(-0.97441764-0.125 * 0.43799073) \\
& =B_{0}^{\text {ext }}-1.02916648 B_{1}^{\text {ext }}=\operatorname{Max}\left\{B^{\text {ext }}\right\}=1.5 \mathrm{~T}
\end{aligned}
$$

Solve for $B_{0}^{\text {ext }}$ and $B_{1}^{\text {ext }}$, we have

$$
B_{0}^{e x t}=0.85 \mathrm{~T}, \quad B_{1}^{\text {ext }}=-0.63157906 \mathrm{~T}, \text { and } B_{2}^{\text {ext }}=0.07894738 \mathrm{~T} .
$$

So the external field is

$$
B^{e x t}=0.85-0.632 \cos (\omega t)+0.079 \sin (2 \omega t) \text {. }
$$

And its derivative is

$$
\frac{\mathrm{d} B^{e x t}}{\mathrm{~d} t}=0.632 \omega \sin (\omega t)+0.158 \omega \cos (2 \omega t) \text {. }
$$




\section{Appendix II. Eddy Current in Spiral Rib}

Fig.II.1 is used to add the argument that the eddy current in spiral rib can be neglected. The applied external magnetic field is perpendicular to the paper.

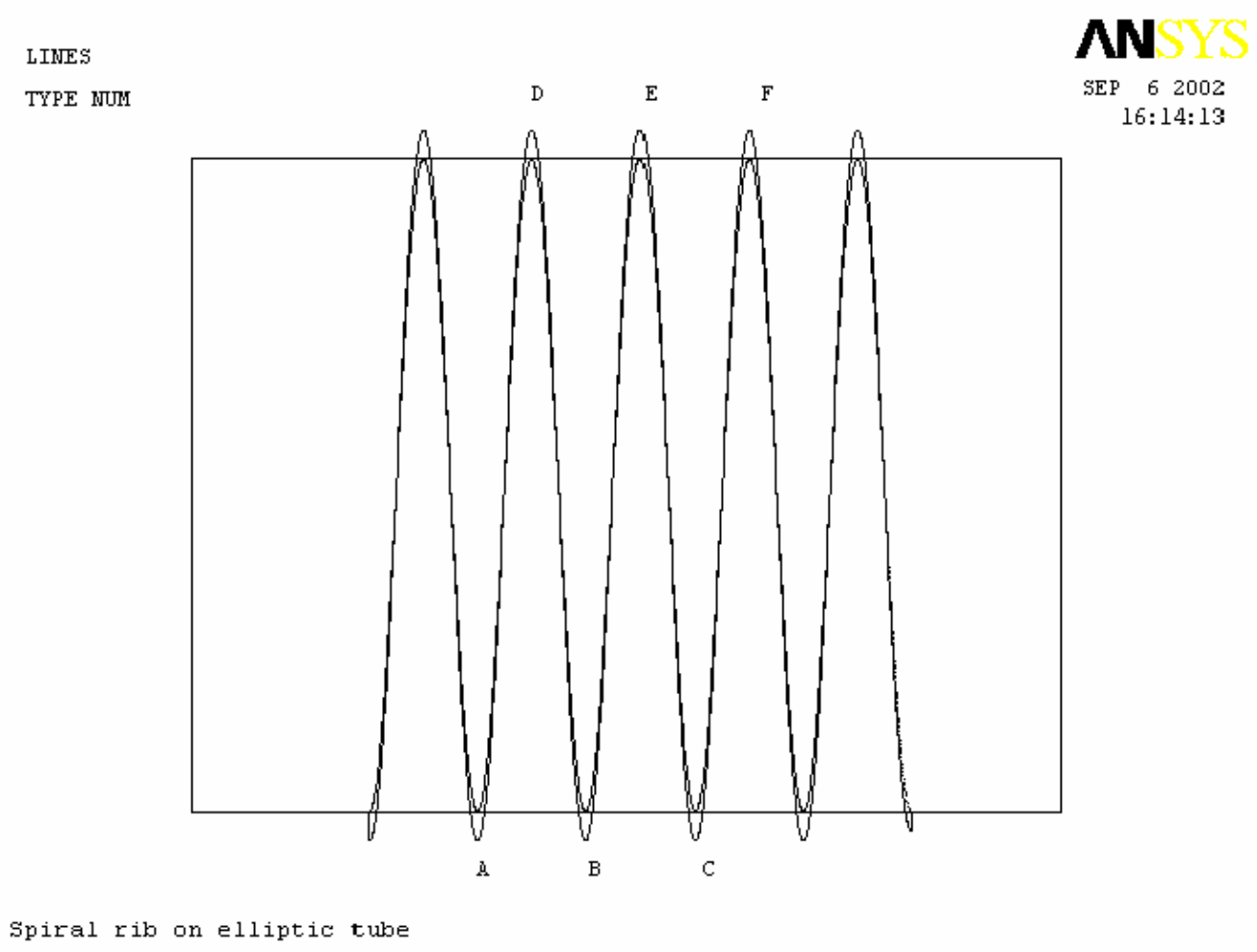

Fig. II.1 Spiral rib on vacuum tube

Since there is only a single rib, it cannot form a closed loop by itself, so part of the tube is needed to close the loop. We assume that the eddy current flows through a closed loop $\mathrm{ABDA}$, and the eddy current is flowing in the anticlockwise direction, hence the eddy current in the segment BD is flowing from B to D. Now consider another close loop DBED. The magnetic flux through this loop is the same as previous loop, so is its changing rate. Therefore the induced electric potential should be the same as in the previous loop. The electric resistance of the two loops is also the same. So we should get same amount of eddy current flow in the same direction, i.e. in anticlockwise direction. For this loop DBED, anticlockwise eddy current means it flow from D to B. So we get two current of the same magnitude flow in different direction in the segment BD, they should cancel out. That means we get no net eddy current in the segment BD.

Similarly, if we consider loop ABED and eddy current in the segment BE, it will be canceled out by the eddy current in the same segment due to loop BCFE.

Therefore we conclude that the should be negligible eddy current in the spiral rib, except at the ends there you cannot find the loop to cancel them. 


\section{Appendix III. Power Loss in Vacuum Tube}

Consider an elliptical vacuum tube with major radius $a$, minor radius $b$ and thickness $c$. A time changing sinusoidal magnetic field $B=B_{0} \sin (\omega t)$ is parallel to its minor axis.

$$
\begin{aligned}
& x=a \cos \theta, \quad y=b \sin \theta, \\
& \mathrm{d} s=\sqrt{(\mathrm{d} x)^{2}+(\mathrm{d} y)^{2}}=\sqrt{(a \sin \theta)^{2}+(b \cos \theta)^{2}} \mathrm{~d} \theta .
\end{aligned}
$$

From Maxwell equations,

$$
\oint \mathbf{E} \cdot \mathrm{d} \mathbf{l}=-\frac{\mathrm{d}}{\mathrm{d} t} \iint_{\mathrm{A}} \mathbf{B} \cdot \mathrm{d} \mathbf{A} \text {. }
$$

Consider a path that locates at $\theta$ with width $\mathrm{d} s$ and length of 1 meter. The return path is at $\pi$ - $\theta$. We have the induced electric voltage and resistance of that 1 meter path $V=x \dot{B}, \quad R=\lambda / c \mathrm{~d} s$. Where $\lambda$ is the resistivity of the tube material.

The power loss then can be calculated as $P=V^{2} / R$,

$\mathrm{d} P=2(x \dot{B})^{2} \frac{c \mathrm{~d} s}{\lambda}$

Since $\dot{B}^{2}=\omega^{2} B_{0}^{2} \cos ^{2}(\omega t)$, and the time average of $\cos ^{2}(\omega t)$ is $1 / 2$, so if we calculate the time averaged power loss then

$$
\begin{aligned}
\bar{P} & =\frac{c \omega^{2} B_{0}^{2}}{\lambda} \int_{-\pi / 2}^{\pi / 2}(a \cos \theta)^{2} \sqrt{(a \sin \theta)^{2}+(b \cos \theta)^{2}} \mathrm{~d} \theta \\
& =\frac{2 c \omega^{2} B_{0}^{2} a^{3}}{\lambda} \int_{0}^{\pi / 2} \cos ^{2} \theta \sqrt{(\sin \theta)^{2}+\left(\frac{b}{a} \cos \theta\right)^{2}} \mathrm{~d} \theta \\
& =\frac{2 c \omega^{2} B_{0}^{2} a^{3}}{\lambda} I
\end{aligned}
$$

Let $u=\sin \theta$, the integration can be written as

$$
I=\int_{0}^{1} \sqrt{\left(1-u^{2}\right)\left\{b^{2} / a^{2}+\left(1-b^{2} / a^{2}\right) u^{2}\right\}} \mathrm{d} u
$$

When $b / a=0, I=1 / 3 ; \quad$ when $\mathrm{b} / a=1, I=\pi / 4$. Numerical integration gives following results. Also listed are the ANSYS finite element results. 


\begin{tabular}{|c|c|c|c|c|c|c|c|}
\hline$b$ (in) & $a$ (in) & $c$ (mil) & $f(\mathrm{~Hz})$ & $B_{0}(\mathrm{~T})$ & $\mathrm{P}(\mathrm{w} / \mathrm{m})$ & $\begin{array}{l}\text { Total } \\
(\mathrm{w} / \mathrm{m})\end{array}$ & $\begin{array}{l}\text { ANSYS } \\
\text { results }\end{array}$ \\
\hline 2 & 3 & 8 & 15 & 0.6316 & 303.8 & & \\
\hline & & & 30 & 0.07895 & 19.0 & 322.8 & 327 \\
\hline 2.5 & 4.5 & 5 & 15 & 0.515 & 384.0 & & \\
\hline & & & 30 & 0.0644 & 24.0 & 408.0 & 411 \\
\hline & & & 15 & 0.6876 & 684.4 & & \\
\hline & & & 30 & 0.08595 & 42.8 & 727.2 & 733 \\
\hline
\end{tabular}

\section{Appendix IV. Electro-magnetic Force on Vacuum Tube}

When the vacuum tube is put into a time changing external magnetic field, eddy current is induced. This eddy current will in turn interact with the external magnetic field. Therefore the vacuum tube will experience a magnetic force.

As in Appendix III, we consider a segment of thickness $c$, width $\mathrm{d} s$ and unit length in $z$ direction. We have a current segment

$$
\mathrm{d} I=\frac{c}{\lambda} x \dot{B} \mathrm{~d} s
$$

This current segment will experience a force of

$$
\mathrm{d} F=\frac{c}{\lambda} x B \dot{B} \mathrm{~d} s
$$

Since $B$ is in $y$ direction, positive $F$ is in the $-x$ direction for $x>0$. If we calculate the total force for $x>0$, we have

$$
F=\frac{c}{\lambda} B \dot{B} \int_{-\pi / 2}^{\pi / 2} x \mathrm{~d} s
$$

The integration can be carried out exactly

$$
\int_{-\pi / 2}^{\pi / 2} x \mathrm{~d} s=2 \int_{0}^{\pi / 2} a \cos \theta \sqrt{(a \sin \theta)^{2}+(b \cos \theta)^{2}} \mathrm{~d} \theta=1.574 a^{2}
$$

Here we have use $b / a=2 / 3$. Now put $c=0.008$ inch, $a=3$ inch, and $\lambda=125 \mathrm{e}-8 \Omega$-m, we have

$$
F=1.462 B \dot{B}
$$

This is a function of time, and its value has the unit of newtons per meter. Figure IV.1 plots its value change with time in one period. The maximum absolute value is $110 \mathrm{~N} / \mathrm{m}$. Compare the force from vacuum pressure

$$
F_{p}=14.5 \mathrm{psi} \times 4 \mathrm{in} \times \frac{39.37 \mathrm{in}}{1 \mathrm{~m}}=2283 \mathrm{lb} / \mathrm{m}=10157 \mathrm{~N} / \mathrm{m}
$$

We see that the magnetic force is only about $1 \%$ of the vacuum pressure force. 


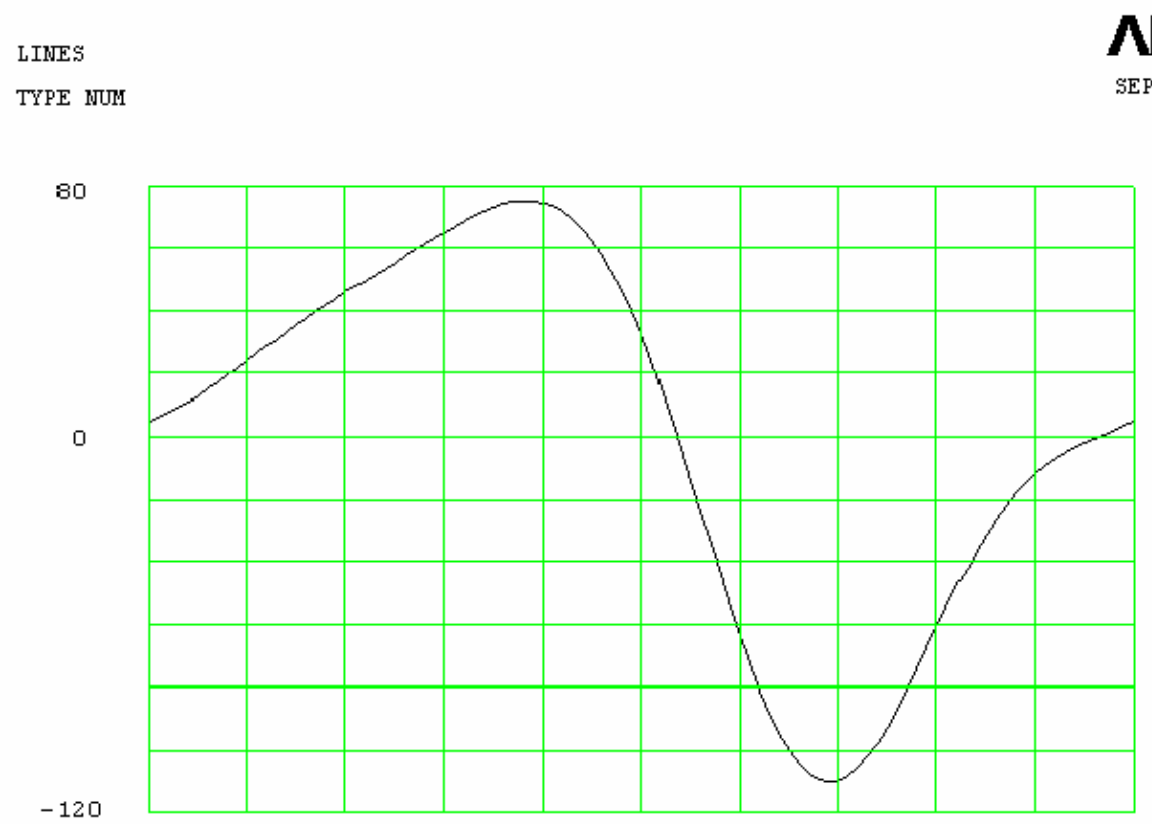

Total magnetic force $(N / m)$ on vacuum tube changes with time

Fig. IV.1 Electro-magnetic force on vacuum tube

\section{Appendix V. Eddy Current Induced Magnetic Field}

Eddy current is produced by changing external magnetic field. This eddy current will produce a magnetic field of its own. When this magnetic field is superimposed onto the external field, the total magnetic field is distorted. Here we estimate the magnitude of this Magnetic filed induced by eddy current.

As in Appendix III, we consider a segment of thickness $c$, width ds. Instead of unit length in $z$ direction, we consider it as infinite. We have a current segment

$$
\mathrm{d} I=\frac{c}{\lambda} x \dot{B} \mathrm{~d} s
$$

This current will produce a magnetic field around it. We consider $y$-component of this magnetic field at the center of the tube (The $x$-component will be canceled out when integrated over entire vacuum tube).

$$
\mathrm{d} B^{*}=\frac{\mu_{0} \cos \theta}{2 \pi \rho} \frac{c}{\lambda} x \dot{B} \mathrm{~d} s, \quad \rho=\sqrt{x^{2}+y^{2}} .
$$

Integrate over entire tube, we have 


$$
\begin{aligned}
B^{*} & =\int_{\theta=0}^{\pi / 2} \frac{2 \mu_{0} \cos \theta}{\pi \rho} \frac{c}{\lambda} x \dot{B} \mathrm{~d} s \\
& =\frac{2 \mu_{0} a c \dot{B}}{\pi \lambda} \int_{0}^{\pi / 2} \sqrt{\frac{(a \sin \theta)^{2}+(b \cos \theta)^{2}}{(a \cos \theta)^{2}+(b \sin \theta)^{2}}} \cos ^{2} \theta \mathrm{d} \theta .
\end{aligned}
$$

The integration is numerically calculated, and its value is 0.657 (for $a / b=1.5$ ). Substitute the numerical values into above equation, we have $B^{*}=6.51 \times 10^{-6} \dot{B}$. Let $B=B_{0} \mathrm{e}^{j \omega t}, \quad \dot{B}=j \omega B_{0} \mathrm{e}^{j \omega t}$, we have

$$
\left|\frac{B^{*}}{B_{0}}\right|=6.51 \times 10^{-6} \omega
$$

Therefore

$$
\begin{aligned}
& \left|\frac{B^{*}}{B_{0}}\right|=0.613 \times 10^{-3}, \quad \text { for } \omega=30 \pi \\
& \left|\frac{B^{*}}{B_{0}}\right|=1.227 \times 10^{-3}, \quad \text { for } \omega=60 \pi
\end{aligned}
$$

\section{Appendix VI. About the ASME Pressure Vessel Code}

( From the ASME pressure vessel code (1995) section VIII division 2.)

AD-140 (c) (page 64) The local primary membrane stress due to any combination of loadings is limited to $1.5 k s_{\mathrm{m}}$. The distance over which stress intensity exceeds $1.1 k$ $s_{\mathrm{m}}$ shall not extend in the meridional direction more than $(R t)^{1 / 2}$.

Table AD 150.1 (page 65) $\quad k=1.0$.

AD-130 (page 63) The design stress intensity value $s_{\mathrm{m}}$ are given in Tables 2A, 2B, 3 and 4 in subpart 1 of section II, part D for vessel materials.

We cannot find the specific material (Inconel 718) in Table 2B. Generally, the stress intensity value is taken as $2 / 3$ of the minimum yield stress of the material. For Inconel 718 , yield stress is $171 \mathrm{ksi}$, so the stress intensity value is $s_{\mathrm{m}}=114 \mathrm{ksi}$.

Limiting stress is $1.5 \mathrm{k} s_{\mathrm{m}}=171 \mathrm{ksi}$. Our model has a maximum stress of $122 \mathrm{ksi}(\mathrm{OK})$. $1.1 \mathrm{k} s_{\mathrm{m}}=125 \mathrm{ksi}$. There is not place the stress reaches this number $(\mathrm{OK})$. 